Revue d'Allemagne et des pays de langue allemande

46-1 | 2014

Les fondements normatifs de l'État constitutionnel moderne en Allemagne. Une approche pluridisciplinaire

\title{
Der Niedergang der FDP
}

\section{Oskar Niedermayer}

\section{(2) OpenEdition \\ Journals}

Édition électronique

URL : https://journals.openedition.org/allemagne/1418

DOI : 10.4000/allemagne.1418

ISSN : 2605-7913

\section{Éditeur}

Société d'études allemandes

Édition imprimée

Date de publication : 30 juin 2014

Pagination : 201-213

ISSN : 0035-0974

Référence électronique

Oskar Niedermayer, "Der Niedergang der FDP“, Revue d'Allemagne et des pays de langue allemande [Online], 46-1 | 2014, Online erschienen am: 29 Juli 2019, abgerufen am 18 Mai 2021. URL: http:// journals.openedition.org/allemagne/1418 ; DOI: https://doi.org/10.4000/allemagne.1418 


\section{Der Niedergang der FDP}

\section{- Oskar Niedermayer*}

\section{Einleitung}

Von der Gründung der Bundesrepublik Deutschland an war die FDP über drei Jahrzehnte lang die unangefochtene „dritte Kraft“ im deutschen Parteiensystem ${ }^{(1)}$. Obwohl sie bei den jeweiligen Bundestagswahlen nur zwischen 6 und 13 Prozent der Wählerstimmen auf sich vereinigen konnte, spielte sie in ihren beiden Funktionen als Mehrheitsbeschafferin und liberales Korrektiv von CDU/CSU und SPD für die Bildung und die Politik der jeweiligen Bundesregierungen eine zentrale Rolle: Mit Ausnahme der CDU/CSU-DP-Regierung von 1957 bis 1961 und der Großen Koalition von Ende 1966 bis 1969 war sie in einer Koalition mit einer der beiden Volksparteien stets an der Regierung beteiligt.

Ihre starke Position änderte sich auch noch nicht mit der Etablierung der Grünen anfangs der 1980er-Jahre, da deren Wählerbasis noch zu gering war und sie von den anderen Parteien als nicht koalitionsfähig angesehen wurden. Mit den zunehmenden Wahlerfolgen und der von der SPD vorangetriebenen koalitionsstrategischen Inklusion der Grünen wurden diese jedoch zur immer stärkeren Konkurrentin um die Schlüsselstellung im Parteiensystem und beendeten 1998 die drei Jahrzehnte lang ununterbrochene Regierungsbeteiligung der Liberalen. Zudem betrat mit der Wiedervereinigung in Gestalt der PDS eine weitere Partei die Bühne, die 1998 zum ersten Mal die Fünf-Prozent-Hürde überwand, bei der Bundestagswahl 2005 sogar die Grünen überflügelte und durch die Vereinigung mit der westdeutschen WASG 2007 unter dem Namen ,Die Linke von einer ostdeutschen Regionalpartei zur gesamtdeutschen Konkurrenz von FDP und Grünen wurde.

* Professor für Politische Wissenschaft (Schwerpunkt Politische Soziologie) an der Freien Universität Berlin.

1 Für eine ausführlichere und auch die Zeit bis zum Frühjahr 2014 einschließende Analyse der FDPEntwicklung vgl. Oskar Niedermayer, „Von der dritten Kraft zur marginalen Partei: Die FDP von 2009 bis nach der Bundestagswahl 2013“, in: Ders. (Hg.), Die Parteien nach der Bundestagswahl 2013, Wiesbaden, Springer VS, 2014 (in Vorbereitung). 
Bestand 2005 eine offene Wettbewerbssituation zwischen den drei kleineren Parteien, so schien die FDP mit ihrem hervorragenden Wahlergebnis bei der Bundestagswahl 2009 und der anschließenden Regierungsübernahme den Grundstein für eine neue Phase als unangefochtene ,dritte Kraft' im Parteiensystem zu legen. Dem Höhenflug von 2009 folgte jedoch 2010 ein jäher Absturz, der sie zur marginalen Partei machte. Bis zum Herbst 2013 konnte sie das Blatt nicht mehr entscheidend wenden und scheiterte bei der Bundestagswahl am 22. September 2013 mit 4,8 Prozent an der Fünf-Prozent-Hürde. Diese Entwicklung soll im Folgenden näher beleuchtet werden.

\section{Der Höhenflug 2009}

Trotz des guten FDP-Ergebnisses konnte 2005 die schwarz-gelbe Wunschkoalition nicht gebildet werden und die Union ging mit der SPD eine Große Koalition ein. In der Folgezeit profilierte sich die FDP unter Guido Westerwelle, der Anfang 2006 neben dem Parteivorsitz auch noch den Fraktionsvorsitz übernahm, als wirtschaftsliberale Reformpartei und eigenständige Alternative zur Unionspolitik in der Regierung. Auch während der im Herbst 2008 einsetzenden weltweiten Finanzmarkt- und Wirtschaftskrise behielt sie diese Ausrichtung bei, während sich die unionsgeführte Bundesregierung zu staatsinterventionistischem Handeln gezwungen sah. Die zur Rettung des Bankensektors als notwendig erachtete (Teil-)Verstaatlichung von Banken und die Diskussionen um eine Staatsbeteiligung an Konzernen wie Opel und Arcandor interpretierte ein Teil der wirtschaftsliberal orientierten Unionsanhänger als Verrat am Markenkern der Union und verlieh ihrem Widerspruch durch die Wahl der FDP Ausdruck, die einerseits eine Koalition mit der Union klar präferierte und andererseits die christdemokratische Abkehr von der sozialen Marktwirtschaft anprangerte.

Inhaltlich setzte die FDP vor der Bundestagswahl und in ihrem Wahlprogramm durchaus auch Akzente beim Thema Bürgerrechte, der Bildungspolitik und in anderen Politikbereichen, in der Außenwahrnehmung dominierte aber das Thema Steuersenkungen mit der Botschaft ,Mehr Netto vom Brutto'. Mit der erheblichen sachpolitischen Kompetenzzuweisung durch die Wähler im Bereich der Steuer- und Wirtschaftspolitik gelang es der Partei, über die liberale Kernklientel hinaus auch Wähler aus anderen Schichten anzusprechen. Dennoch wurde die FDP auch 2009 mit dem besten Wahlergebnis ihrer Geschichte (14,6\%) nicht zu einer Partei für das ganze Wahlvolk, sondern behielt ihre Schwerpunkte bei den etablierten Leistungsträgern mit hoher formaler Bildung bei. Der Wahltriumph, flankiert durch Erfolge auf der Kommunal- und Landesebene seit $2006^{(2)}$, ließ sie jedoch wieder als unangefochtene dritte Kraft im Parteiensystem erscheinen.

2 Bei allen Kommunalwahlen ab 2006 außer in Bayern erzielte die FDP im Landesdurchschnitt Werte von mehr als 5 Prozent. In der ersten Jahreshälfte 2006 war die FDP in 11 Landtagen vertreten und an 3 Landesregierungen beteiligt. In der zweiten Jahreshälfte 2009 war sie außer in Hamburg in allen Bundesländern parlamentarisch repräsentiert und an 8 Landesregierungen beteiligt. 


\section{Der Absturz 2010 und die Entwicklung bis zur Bundestagswahl}

Die sich im Bundestagswahlergebnis ausdrückende hohe Unterstützung von CDU/ CSU und FDP durch die Bürgerinnen und Bürger währte nicht allzu lange. Hatte die Vorgängerregierung aus Union und SPD kurz vor der Bundestagswahl in der Bewertung durch die Bevölkerung noch verhalten positiv abgeschlossen, so zeigte schon die erste Messung der Zufriedenheit mit der Arbeit der neuen Bundesregierung zwei Monate nach der Wahl einen Rückgang, und in den Folgemonaten stürzte sie deutlich ab.

Analysen der langfristigen Entwicklung der Zufriedenheit der Bevölkerung mit der Arbeit der jeweiligen Bundesregierung zeigen jedoch, dass das Schicksal der schwarz-gelben Koalition keine Ausnahmeerscheinung ist. In der Regel unterliegen Regierungen im Laufe der Legislaturperiode einem ,Popularitätszyklus'. Sie haben vor der Wahl bei ihren Anhängern hohe Erwartungen geweckt, die sie durch ihre konkrete Politik meist enttäuschen, was in der ersten Hälfte der Legislaturperiode zu deutlichen Popularitätseinbußen führt. Einen wesentlichen Unterschied der jetzigen Regierungskoalition zu den verschiedenen Vorgängerregierungen gibt es jedoch: die Bewertung des kleinen Koalitionspartners ${ }^{(3)}$. Die Verschlechterung der Bewertung der Regierungsarbeit der Union 2010 hielt sich noch im Rahmen, und sie konnte schon ab Anfang 2011 wieder positive Werte verbuchen, die sich ab Herbst 2011 kontinuierlich steigerten und kurz vor der Wahl 2013 ihren Höhepunkt erreichten. Die FDP jedoch sackte von einem nur leicht negativen Wert kurz nach der Bundestagswahl im Sommer 2010 auf einen Minusrekord ab. Schlechter wurde die Arbeit einer Regierungspartei in den mehr als 30 Jahren der Messung von Regierungszufriedenheit noch nie bewertet. Zwar konnte die Partei ab Anfang 2012 ihre Werte bis zur heißen Phase des Wahlkampfs wieder kontinuierlich steigern, sie blieben aber im negativen Bereich und gingen kurz vor der Wahl wieder leicht nach unten.

Die dramatische Verschlechterung der Bewertung der Regierungsarbeit der FDP schlug sich auch deutlich in der politischen Stimmung der Bevölkerung nieder. Die Partei verlor innerhalb von acht Monaten fast vier Fünftel ihrer Wählerklientel und lag in der politischen Stimmung von Mai 2010 bis zum Jahresende bei 3-4 Prozent, ein in der deutschen Parteiengeschichte einmaliger Absturz einer Regierungspartei. War sie aus der Bundestagswahl als eindeutige ,dritte Kraft' im Parteiensystem hervorgegangen, so wurde sie jetzt zur marginalen Partei, die nicht nur deutlich hinter den 2010 einen noch nie dagewesenen Höhenflug erlebenden Grünen lagen, sondern auch hinter der Linkspartei, obwohl diese selbst einen Unterstützungsrückgang zu verkraften hatte. In den nächsten zweieinhalb Jahren verschlechterte sich die Situation noch: Die Stimmungswerte lagen nur noch zwischen 2 und 3 Prozent und fielen zweimal sogar auf den absoluten historischen Tiefststand von 1 Prozent. In den letzten drei Monaten vor der Wahl verbesserte sich die Lage etwas und kurz vor der Wahl lagen die Projektionen aller Institute für das Abschneiden der FDP bei 5-6 Prozent, sodass man davon ausging, die Partei würde es ganz knapp schaffen, im Bundestag zu verbleiben. Am Ende fehlten ihr jedoch 0,2 Prozent.

3 Zur Bewertung der Arbeit der Bundesregierung und der sie tragenden Parteien vgl. Forschungsgruppe Wahlen e.V., Politbarometer (div. Ausgaben). 


\section{Die inhaltlichen und personellen Gründe für den Absturz 2010}

Für den Absturz der FDP nach der Bundestagswahl 2009 sind sowohl inhaltliche als auch personelle Faktoren verantwortlich, die dadurch eng verwoben sind, dass der Vorsitzende Guido Westerwelle „die FDP zur Einmannpartei machte“(4). Westerwelle legte die FDP vor der Wahl in der Außenkommunikation fast ausschließlich auf die Positionierung als Steuersenkungspartei fest. Damit wurden - über die Kernklientel der FDP hinaus - sehr hohe Erwartungen geweckt, die nach der Wahl enttäuscht wurden. Der tiefere Grund hierfür liegt in der Tatsache, dass die drei vermeintlichen ,Traumpartner' CDU, CSU und FDP von Anfang an weit weniger inhaltliche Gemeinsamkeiten aufwiesen als gemeinhin angenommen wurde. Man kann sogar die These aufstellen, dass Schwarz-Gelb eine ,historisch verspätete Koalition darstellte. Zur Bundestagswahl 2005 hatten CDU, CSU und FDP ein klares gemeinsames Projekt, nämlich eine an marktfreiheitlichen Prinzipien ausgerichtete Reform, während die drei Parteien 2009 im wirtschafts- und finanzpolitischen Bereich deutlich unterschiedliche Positionen vertraten. Insbesondere stand die CDU den weit gehenden Steuersenkungsforderungen der FDP (aber auch der CSU) sehr skeptisch gegenüber und legte angesichts der noch nicht ausgestandenen Wirtschaftskrise und der hohen Staatsverschuldung mehr Wert auf Haushaltskonsolidierung. Im Koalitionsvertrag wurde dann der FDP/CSU-Forderung nach Steuersenkungen in Milliardenhöhe entsprochen aber gleichzeitig ein Finanzierungsvorbehalt im Sinne der CDU aufgenommen. Damit war die Grundlage für einen Dauerstreit der Koalitionspartner gelegt.

Der einzige Sieg der FDP in diesem Dauerstreit war die - zusammen mit der CSU Ende 2009 im Rahmen des ersten größeren Gesetzesvorhabens (Wachstumsbeschleunigungsgesetz) der CDU abgerungene Halbierung des Mehrwertsteuersatzes für das Hotelgewerbe. Der Sieg geriet jedoch zum Desaster, als Mitte Januar 2010 mehrere im Umfeld der Bundestagswahl erfolgte großzügige FDP-Spenden der Familie Finck, Haupteigentümer der Möwenpick-Hotelgruppe, bekannt wurden. Dies heftete der FDP in den Augen der Wähler wieder das Image der ,Partei der Besserverdienenden' an, die statt des versprochenen, mehr Netto vom Brutto' für die breite Masse der Bevölkerung nur Verbesserungen für ihre Kernklientel durchsetzt. Zudem wurde die FDP zur ,Nullthemenpartei', als Kanzlerin Angela Merkel nach der Landtagswahl in Nordrhein-Westfalen im Mai 2010 und unter dem Eindruck der Eurokrise Steuersenkungen eine klare Absage erteilte.

Die Kompetenzzuschreibungen durch die Bevölkerung im Bereich des ,Markenkerns' der FDP, der Steuer- und Wirtschaftspolitik, verschlechterten sich daraufhin dramatisch: Hatten kurz vor der Bundestagswahl 19 bzw. 14 Prozent der Wähler von allen Parteien am ehesten der FDP zugetraut, eine gute Steuerpolitik zu betreiben bzw. die Wirtschaft in Deutschland voranzubringen, so waren Ende 2010 nur noch 5 bzw. 4 Prozent dieser Meinung.

Einen wesentlichen Anteil am FDP-Absturz hatte zudem die Tatsache, dass ihr Vorsitzender Westerwelle den Rollenwechsel vom aggressiven Oppositionspolitiker zum

4 So der Politikwissenschaftler Jürgen Dittberner, „Vom Erfolg betrunken“, Der Tagesspiegel vom 21. Januar 2010. 
deutschen Außenminister nicht wirklich vollzog und dadurch den ,Amtsbonus nicht für sich nutzen konnte, den diese Rolle bei der Bewertung durch die Bevölkerung mit sich bringt. Guido Westerwelle wurde von der Bevölkerung nie sonderlich gut beurteilt. Einen persönlichen Spitzenwert erreichte er kurz nach der Bundestagswahl 2009, stürzte dann aber dramatisch ab und verharrte das gesamte Jahr 2010 über bei Negativwerten, wie sie in den mehr als 30 Jahren der Messung von Politikerbewertungen noch nie ein Außenminister hinnehmen musste ${ }^{(5)}$.

Ursächlich hierfür ist vor allem die Tatsache, dass die Bevölkerung von einem Außenminister erwartet, staatstragend über den Niederungen der innenpolitischen Konflikte zu schweben. Westerwelle kehrte jedoch schon in der ersten Februarhälfte 2010 durch provokante Äußerungen in der Debatte um die Angemessenheit der Hartz IV-Sätze aus den Sphären der Diplomatie in die Innen- und Parteipolitik zurück. Zudem wurde ihm Anfang März der Vorwurf der Verquickung von dienstlichen, parteipolitischen und privaten Verbindungen auf seinen Dienstreisen gemacht.

Da der dramatische Ansehensverlust ihres Vorsitzenden der FDP immer mehr schadete, wurde die innerparteiliche Kritik an ihm ab dem Sommer 2010 immer lauter. Zudem war auch die Bevölkerung zu fast drei Vierteln der Ansicht, Westerwelle habe in der letzten Zeit seiner Partei mehr geschadet als genutzt ${ }^{(6)}$. Ausgelöst durch ein Interview des schleswig-holsteinischen Fraktionsvorsitzenden Wolfgang Kubicki, in dem er die Lage der FDP als fast aussichtslos bezeichnete, kam es schließlich im Dezember 2010 zu einer von FDP-Funktionären immer wieder befeuerten Mediendiskussion um die Ablösung des Vorsitzenden. Der innerparteiliche Aufstand versandete jedoch wieder, nachdem Westerwelle zum freiwilligen Verzicht auf den Parteivorsitz nicht bereit war und aufgrund inhaltlicher wie persönlicher Differenzen in den Reihen des Führungspersonals keine einheitliche gegnerische Front mit einer klaren personellen Alternative gebildet werden konnte: Junge Spitzenpolitiker wie der Generalsekretär Christian Lindner, der nordrhein-westfälische Landeschef Daniel Bahr oder der Gesundheitsminister Philipp Rösler wollten ihre Parteikarriere nicht durch die Übernahme der Rolle des Königsmörders gefährden und ältere Spitzenfunktionäre wie der Wirtschaftsminister Rainer Brüderle sahen sich nicht als Übergangslösung. Zudem bestanden in den Reihen der Kritiker deutlich unterschiedliche Auffassungen über den inhaltlichen Kurs, den die FDP zukünftig einschlagen sollte.

\section{Der Versuch des personellen und inhaltlichen Neubeginns}

Anfang 2011 lag die FDP am Boden. Daran änderte auch die Rede des Vorsitzenden auf dem traditionellen Dreikönigstreffen der Partei nichts, die innerparteilich auf ein geteiltes Echo stieß und von den Medien verhalten bis negativ kommentiert wurde. In der zweiten Februarhälfte schien sich jedoch eine leichte Erholung abzuzeichnen. Zwar verblieb die Partei in der politischen Stimmung auf ihren geringen Werten, die Zufriedenheit mit ihrer Regierungsarbeit stieg jedoch etwas an und ihr Vorsitzender begann in den Augen der Medien und der Bürger an Statur zu gewinnen, als er die

5 Vgl. Forschungsgruppe Wahlen e.V., Politbarometer (div. Ausgaben).

6 Vgl. Infratest dimap, DeutschlandTREND, Juni 2010. 
Umwälzungsprozesse in der nordafrikanischen Krisenregion geschickt als Bühne zur Profilierung als seriöser Außenminister nutzte. Zudem wurde der Wiedereinzug in die Hamburger Bürgerschaft bei der Wahl vom 20. Februar als Beleg für eine Konsolidierung der Partei auch auf Bundesebene gedeutet, obwohl das dortige Abschneiden primär auf landespolitische Faktoren zurückzuführen war.

In der ersten Märzhälfte machten die Partei und ihr Vorsitzender die Ansätze für eine Verbesserung der Lage jedoch wieder zunichte. Die deutsche Enthaltung im UN-Sicherheitsrat bei der Resolution über die Einrichtung einer Flugverbotszone über Libyen verärgerte massiv die wichtigen Bündnispartner, brachte vor allem die Beziehungen zu Frankreich auf einen neuen Tiefpunkt und führte zu der Befürchtung, Außenminister Westerwelle sei dabei, das die feste Einbindung in den Westen als Fundament der deutschen Außenpolitik aufzulösen.

Weit folgenreicher für die Lage der FDP war jedoch die Reaktion der Bundesregierung auf die Reaktorkatastrophe, die sich am 11. März im Gefolge eines Erdbebens und Tsunamis im japanischen Fukushima ereignete. Durch das spezifische ,Framing der Katastrophe in den deutschen Medien stark befördert, hielt fast die Hälfte der Bevölkerung, auch die deutschen Kernkraftwerke für eher unsicher und befürchtete, dass es in Deutschland auch einmal zu einem schweren Atomunfall kommen könnte $^{(7)}$. Die Bundesregierung sah sich daher zur politischen Reaktion gezwungen und verkündete eine abrupte Kehrtwende in der Atompolitik mit der dreimonatigen Aussetzung der erst vor einem halben Jahr gegen große Widerstände beschlossenen Verlängerung der Laufzeit der Atomkraftwerke um durchschnittlich 12 Jahre und der vorläufigen Abschaltung von sieben älteren Atommeilern. Der Kurswechsel konnte den Wählern in der kurzen Zeit bis zu den Landtagswahlen in Sachsen-Anhalt am 20. März und in Baden-Württemberg und Rheinland-Pfalz am 27. März jedoch nicht glaubhaft vermittelt werden.

Die FDP geriet dabei in ein besonders großes Glaubwürdigkeitsdefizit, da sie die treibende Kraft hinter der Laufzeitverlängerung gewesen war und sich einerseits ihr Vorsitzender jetzt plötzlich als überzeugter Atomkraftgegner gab und andererseits ihr Wirtschaftsminister Rainer Brüderle nach Medienberichten das AKW-Moratorium in einer Sitzung des Bundesverbands der Deutschen Industrie als Wahlkampftaktik bezeichnete. Die Wahlergebnisse der FDP waren dann auch entsprechend: in SachsenAnhalt und Rheinland-Pfalz verfehlte sie den Einzug ins Parlament und in BadenWürttemberg erhielt sie nur noch halb so viel Zustimmung wie bei der letzten Wahl. Guido Westerwelle ließ noch am Wahlabend verkünden, er werde auf keinen Fall von seinen Ämtern zurücktreten, aber angesichts des Wahldesasters war die parteiinterne und mediale Diskussion um seine Ablösung nicht mehr zu stoppen. Eine Woche später gab er bekannt, beim Parteitag im Mai 2011 nicht mehr für das Amt des Parteivorsitzenden kandidieren zu wollen.

Die FDP-Führung einigte sich relativ schnell darauf, dass Gesundheitsminister Philipp Rösler der neue FDP-Vorsitzende werden sollte. Anschließend erfolgte jedoch ein wochenlanger Machtkampf um einen weiteren Umbau der Partei- und Fraktionsführung, der in der Partei und der Bevölkerung deutliche Zweifel an den

7 Vgl. Forschungsgruppe Wahlen e.V., Politbarometer extra vom 14. März 2011. 
Führungsqualitäten des designierten neuen Vorsitzenden weckte ${ }^{(8)}$ und erst kurz vor dem Parteitag zumindest in großen Teilen zugunsten seiner personellen Vorstellungen entschieden wurde. Der seit der AKW-Affäre und dem Scheitern des von ihm geführten rheinland-pfälzischen Landesverbands bei der Landtagswahl angeschlagene Wirtschaftsminister Rainer Brüderle räumte seinen Posten zugunsten von Philipp Rösler. Brüderle wurde Nachfolger der wegen ihrer schlechten Außenwirkung viel kritisierten Fraktionsvorsitzenden Birgit Homburger, der wiederum der Verzicht auf ihr Amt nur abgerungen werden konnte, weil man ihr den Posten einer der drei stellvertretenden Parteivorsitzenden versprach. Der bisherige Staatssekretär im Gesundheitsministerium, Daniel Bahr, übernahm den Ministerposten und verzichtete dafür, wie auch Brüderle, auf einen Stellvertreterposten in der Parteiführung. Durch diesen personellen Umbau wollte sich der neue Parteivorsitzende in Zukunft im Bereich des Markenkerns der FDP, der Wirtschaftspolitik, mit positiven Botschaften bei den Wählern profilieren.

Allerdings erklärte man auf dem Bundesparteitag am 13. Mai 2011 die Diskussion um den Verbleib von Guido Westerwelle im Außenamt für beendet, so dass der von den Wählern immer noch sehr schlecht bewertete Politiker weiterhin das Bild der FDP nach außen prägte. Hinzu kam, dass der mit 95,1 Prozent gewählte neue Vorsitzende Philipp Rösler die Wähler von Anfang an nicht überzeugen konnte ${ }^{(9)}$. Er startete mit einer leicht negativen Beurteilung, konnte sich kurzfristig etwas steigern und stürzte dann im Urteil der Deutschen im nächsten halben Jahr so stark ab, dass er ab Anfang 2012 schlechter bewertet wurde als Westerwelle. Danach blieb er deutlich im negativen Bereich und Mitte August 2012 wurde er gar nicht mehr zu den 10 wichtigsten Politikern gezählt. Damit war erstmals seit über 11 Jahren der Parteivorsitzende der FDP in den Augen der Bevölkerung nicht mehr unter den Spitzenpolitikern vertreten. Erst Ende November kehrte er in die Spitzengruppe zurück, fiel jedoch von Mitte Mai bis Ende Juni 2013 und sogar kurz vor der Wahl wieder heraus.

Ein wesentlicher Grund für die schlechte Beurteilung Röslers war die Tatsache, dass er das in seiner Rede auf dem Parteitag gegebene Versprechen, „ab heute wird die FDP liefern“, also eine im liberalen Wertesystem verwurzelte Antwort auf gesellschaftliche Problemlagen entwickeln, in konkrete politische Ziele umsetzen und diese im Rahmen der Regierungspolitik verwirklichen, in den Augen der Bürgerinnen und Bürger in der Folgezeit nicht erfüllte. Schon der erste Profilierungsversuch, das Ausstiegstempo aus der Atomenergie aus ökonomischen Gründen zu drosseln, geriet zur Bruchlandung, da die Union auf den - sich auch noch zerstritten präsentierenden - Koalitionspartner keinerlei Rücksicht nahm. Da das Koalitionsklima daraufhin immer schlechter wurde, kam die Union der traditionellen FDP-Forderung entgegen und schnürte ein vages Steuersenkungspaket für Anfang 2013, dessen Realisierungschancen aber bei der Bevölkerung auf größte Skepsis stießen. Die 100-Tage-Bilanz des neuen Parteichefs fiel daher in den Medien deutlich negativ aus.

8 Nach einer Umfrage des Meinungsforschungsinstituts Emnid von Anfang Mai trauten vier Fünftel der Deutschen Rösler nicht zu, die FDP aus der Krise zu führen.

9 Zur Bewertung Röslers und Westerwelles vgl. Forschungsgruppe Wahlen e.V., Politbarometer (div. Ausgaben). 
Hinzu kam, dass Guido Westerwelle nach seinem verunglückten Agieren zum NATO-Einsatz in Libyen im Herbst 2011 neue Minusrekorde in der Bevölkerungsbewertung aufstellte und wieder eine Ablösungsdebatte aufkam. Verschärft wurde die erneute Krise noch durch die Wahlschlappen bei den Landtagswahlen in Mecklenburg-Vorpommern und Berlin im September, wo die Partei beide Male etwa drei Viertel ihrer Wähler verlor und aus den Landtagen flog. Kurz vor der Wahl in Berlin hatte man noch versucht, mit einer harten Haltung zur europäischen Verschuldungskrise Ressentiments gegen die Griechenlandhilfen zu bedienen - Rösler selbst brachte eine geordnete Insolvenz Griechenlands ins Spiel. Damit konnte man das Wahldesaster nicht abwenden, beschwor aber eine neue Koalitionskrise herauf und musste sich nun innerparteilich einer Gruppe von marktliberal motivierten Eurokritikern unter der Führung des Abgeordneten Frank Schäffler erwehren, die einen Mitgliederentscheid über die Haltung der FDP zur von Kanzlerin Angela Merkel befürworteten Einrichtung des unbefristeten europäischen Stabilitätsmechanismus (ESM) durchsetzte. Da man bei einem Erfolg des Mitgliederentscheids den Bruch der Regierungskoalition befürchtete, wandte sich die Parteiführung auf dem Sonderparteitag am 13. November, auf dem neben der Bildungspolitik und der Haltung zum Mindestlohn auch über den Euro-Stabilitätspakt diskutiert wurde, massiv gegen die innerparteilichen Kritiker des Eurorettungskurses.

Noch bevor das Ergebnis des Mitgliederentscheids feststand, trat der zu den jungen Hoffnungsträgern der Partei gezählte Generalsekretär Christian Lindner zurück. Damit sahen viele der Kommentatoren auch die programmatische Neuausrichtung der FDP gescheitert und Rösler wurde ein baldiges politisches Ende vorhergesagt. Das Scheitern des Mitgliederentscheids wegen Nichterfüllung des notwendigen Quorums rettete ihn jedoch. Als er beim traditionellen Dreikönigstreffen der Partei Anfang Januar 2012 mit seiner Rede, in der er vor allem auf den Begriff, Wachstum 'als zukünftigen identitätsstiftenden Terminus der FDP setzte, wieder kein Aufbruchssignal setzen konnte, machten neue Putschgerüchte die Runde. Als zukünftiger Parteivorsitzender wurde Rainer Brüderle gehandelt, der sich in der Rolle als Fraktionsvorsitzender als neuer starker Mann der FDP profiliert hatte. Als Rösler jedoch in der Frage des am 18. März 2012 zu wählenden neuen Bundespräsidenten eine Machtprobe mit Angela Merkel riskierte und die FDP auf den auch von SPD und Grünen unterstützten Joachim Gauck festlegte, gewann er in den Augen seiner Kritiker wieder an Statur. Allerdings verlor er sie sofort wieder, als er sich nach der Wahl in aller Öffentlichkeit über Merkels Agieren lustig machte und die Landtagswahl im Saarland am 25. März mit einer dramatischen Niederlage und dem erneuten parlamentarischen Repräsentationsverlust endete. Kurz darauf hielten drei Fünftel der Bevölkerung die FDP für verzichtbar, nur 35 Prozent waren der Ansicht, sie würde als Partei noch gebraucht, und drei Fünftel glaubten nicht, dass Rösler am Jahresende noch FDP-Vorsitzender sein würde ${ }^{(10)}$.

Die auch in der Partei vorhandene Skepsis bezüglich des Schicksals ihres Vorsitzenden speiste sich vor allem aus der Erwartung, die FDP würde die anstehenden Landtagswahlen in Schleswig-Holstein sowie in Nordrhein-Westfalen in der ersten Maihälfte 
2012 auch verlieren und Rösler sei danach als Vorsitzender nicht mehr tragbar. Nahrung bekam diese Skepsis im April durch einen erbitterten Streit zwischen Rösler, Westerwelle und dem schleswig-holsteinischen Rösler-Kritiker Wolfgang Kubicki um die inhaltlich-programmatische Ausrichtung der Partei und ein schwaches Auftreten Röslers auf dem Bundesparteitag, auf dem die FPD ein neues Grundsatzprogramm beschloss. Mit einer sich explizit von der Bundespartei und ihrem Vorsitzenden distanzierenden Strategie konnten jedoch die beiden Landesverbände mit Wolfgang Kubicki in Schleswig-Holstein an der Spitze wenigstens den Verbleib im Landtag sichern und mit Christian Lindner als Spitzenkandidat in Nordrhein-Westfalen sogar in der Wählergunst zulegen. Rösler interpretierte dies als gemeinsamen Erfolg, und die innerparteilichen Kritiker, die vor der NRW-Wahl nach Medieninformationen bereits ein konkretes Szenario zu Röslers Ablösung entworfen hatten, wagten den Putsch wieder nicht. Allerdings ging der Autoritätsverlust Röslers jetzt so weit, dass Parteifreunde ihn immer offener kritisierten und ihm bei der Wahlkampagne zur Bundestagswahl nur eine Nebenrolle zugestehen wollten. Über die Ausrichtung der Kampagne gab es zudem schon im August 2012 Streit zwischen dem Vorsitzenden, der die FDP in Abgrenzung zu SPD und Grünen in traditioneller Weise als marktliberale Partei positionieren wollte, und Wolfgang Kubicki, der eine Öffnung in Richtung eines Ampelbündnisses propagierte.

Anfang September gab es dann erneuten Streit, diesmal um den schlingernden EuroKurs des Vorsitzenden. Zum Jahresende hin wurde immer deutlicher, dass der Fraktionsvorsitzende Rainer Brüderle zum heimlichen FDP-Chef aufgestiegen war, weil er berechenbar blieb, während Rösler in vielen Fragen einen überraschenden Kurswechsel nach dem anderen vollzogen hatte. Ende Dezember versuchte Rösler, der Partei mit einem aus wirtschaftsliberalen Glaubenssätzen - u.a. der Ablehnung eines Mindestlohns - bestehenden, auf die Mobilisierung der Kernklientel setzenden Positionspapier wieder eine Richtung vorzugeben. Es schien in der Partei jedoch ausgemacht zu sein, dass man ihn bei einem Scheitern der FDP bei der Landtagswahl in Niedersachsen im Januar 2013 stürzen würde. Dies wurde umso wahrscheinlicher, als der Machtkampf zwischen Rösler und seinen Kritikern kurz vor der Wahl erneut eskalierte und Entwicklungsminister Dirk Niebel auf dem Dreikönigstreffen der Partei in seiner Rede eine schnellstmögliche Neuaufstellung der Führungsmannschaft forderte. Da sich die niedersächsische CDU jedoch nur bei einem Verbleib der mit einer schwachen Leistungsbilanz und geringen Kompetenzwerten kämpfenden FDP im Landtag Chancen auf eine Fortsetzung der CDU-geführten Landesregierung ausrechnete, beförderte sie den Versuch der FDP, sich durch taktische ,Leihstimmen' CDU-naher Wähler vor der Niederlage zu retten. Diese Strategie hatte einen unerwartet großen Erfolg: Statt eine drastische Niederlage einstecken zu müssen, konnte die FDP durch den Zuwachs von über 100.000 Stimmen von ehemaligen CDU-Wählern ${ }^{(11)}$ ihren Stimmenanteil sogar steigern, während die CDU 6,5 Prozentpunkte verlor und die Mandatsmehrheit von schwarz-gelb im Landtag knapp verfehlt wurde.

11 Vgl. Infratest dimap, Wahlreport. Landtagswahl Niedersachsen 2013, Berlin, 2013, S. 14. 


\section{Der Wahlkampf zur Bundestagswahl}

Obwohl der Wahlerfolg der FDP in Niedersachsen nicht aus eigener Kraft erreicht wurde, sondern eindeutig auf Leihstimmen von CDU-nahen Wählern zurückzuführen war ${ }^{(12)}$, stärkte der Wahlausgang die Stellung Philipp Röslers. Der nutzte die Gunst der Stunde und bot sofort nach der Wahl seinem Widersacher Rainer Brüderle kaltblütig an, nicht nur Spitzenkandidat für die Bundestagswahl, sondern auch Parteivorsitzender zu werden, wobei er kalkulierte, dass Brüderle der Mut fehlen würde, kurz nach einer gewonnenen Wahl den ,Königsmörder ${ }^{`}$ zu spielen. Mit dieser Einschätzung lag er richtig, und daher beschloss die FDP-Führung am 21 Januar, mit einer Doppelspitze aus dem Spitzenkandidat Brüderle und dem Parteivorsitzenden Rösler in den Bundestagswahlkampf zu ziehen. Die Wähler betrachteten diese Personalentscheidung allerdings sehr skeptisch. Kurz nach seiner Nominierung durch den Parteivorstand wurden zudem durch eine Journalistin der Vorwurf gegen Brüderle erhoben, ihr gegenüber an einer Hotelbar anzügliche Bemerkungen gemacht zu haben, was eine breite öffentliche Sexismusdebatte auslöste und dem Ansehen Brüderles schadete. Die Partei schloss jedoch die Reihen, zeigte sich mit Brüderle solidarisch und wählte ihn auf dem der Neuwahl der Parteiführung gewidmeten Sonderparteitag am 9. März 2013 per Akklamation zum Spitzenkandidaten. Ansonsten verteilte die Parteibasis die Machtgewichte in der Parteispitze neu. Rösler wurde erwartungsgemäß wiedergewählt, einer der neuen Stellvertreter wurde Christian Lindner. Zudem wurde mit Wolfgang Kubicki auch der zweite erfolgreiche Wahlkämpfer als Beisitzer in das Führungsgremium aufgenommen und der Rösler-Kritiker Dirk Niebel fiel durch.

Inhaltlich sendete die FDP-Führung in der Vorbereitung des Wahlprogramms bezüglich ihrer Positionierung im Sozialstaatskonflikt zwischen sozialer Gerechtigkeit und Marktfreiheit teilweise widersprüchliche Signale aus: Einerseits hielt man bei der Euro-Rettung marktfreiheitliche Prinzipien hoch, wandte sich daher gegen jegliche Form von Euro-Bonds und die Einführung einer Finanztransaktionssteuer und forderte innenpolitisch den Abbau der ,kalten Progression' im Einkommensteuertarif sowie die schrittweise Abschaffung des Anfang der 1990er-Jahre als befristete Sondersteuer eingeführten Solidaritätszuschlags. Andererseits wurde statt Steuersenkungen nun die Haushaltskonsolidierung mit oberster Priorität versehen, und vor allem wurde mit der Formel von der „fairen Entlohnung“ und von der Leistungsgerechtigkeit auch am unteren Ende der Lohnskala der Weg für eine branchenspezifische und regionale Ausweitung von Lohnuntergrenzen geebnet, die Rösler noch ein paar Wochen zuvor vehement abgelehnt hatte. Damit versuchte man, sich dem gesellschaftlichen mainstream anzupassen und das Image der sozialen Kälte loszuwerden. Mit dem Beschluss $\mathrm{zu}$ den Lohnuntergrenzen verlor man jedoch ein Alleinstellungsmerkmal im Parteiensystem, das für die liberale Kernklientel aus dem Mittelstand zentral war. Auf der zweiten zentralen Konfliktlinien des deutschen Parteienwettbewerbs, dem Konflikt zwischen progressiv-libertären und konservativ-autoritären Werten, wollte man sich mit der Ablehnung schärferer Sicherheitsgesetze, der Zulassung der doppelten Staatsbürgerschaft und der Durchsetzung der generellen rechtlichen Gleichstellung

12 Für 80 Prozent der FDP-Wähler hieß die präferierte Partei eigentlich CDU (vgl. Forschungsgruppe Wahlen e.V., Kurzanalyse der Landtagswahl in Niedersachsen). 
gleichgeschlechtlicher eingetragener Lebenspartnerschaften mit der traditionellen Ehe wieder deutlicher progressiv-libertär positionieren. Auf dem Programmparteitag am 4. Mai wurde diese Positionierung der Partei - nach zum Teil kontroverser Diskussion im Sinne der Parteiführung beschlossen. Weder die personellen noch die inhaltlichen Veränderungen halfen der FDP jedoch, aus dem Umfragetief herauszukommen: Sie blieb das gesamte erste Wahlhalbjahr bei Stimmungswerten von 2 bis 3 Prozent.

Hinzu kam, dass der wegen der Sexismusaffäre schon angeschlagene Spitzenkandidat Rainer Brüderle wegen eines Sturzes mit Knochenbrüchen von Mitte Juni bis Mitte August für den Wahlkampf ausfiel. Auch nach seiner Genesung schaffte es Brüderle nicht, über die Kernklientel der FDP hinaus bei den Wählern zu punkten: Er wurde den gesamten Wahlkampf über von den Bürgern nicht zu den wichtigsten Politikern gezählt, mit seiner Arbeit waren kurz vor der Wahl zwei Drittel der Bürger nicht zufrieden und er erhielt die schlechteste generelle Bewertung der Spitzenkandidaten aller Parteien. Dem Parteivorsitzenden Philipp Rösler ging es jedoch nicht besser, im Gegenteil: Mit seiner Arbeit waren kurz vor der Wahl sogar 80 Prozent der Wähler nicht zufrieden und seine generelle Bewertung war noch schlechter als die von Brüderle ${ }^{(13)}$. Daher schien Guido Westerwelle immer mehr zum personellen Zugpferd der Kampagne zu werden. Seine Beurteilung durch die Bevölkerung hatte sich seit Anfang 2012 mit seiner Konzentration auf seine Rolle als Außenminister und der Vermeidung weiterer Fehler kontinuierlich verbessert. Er setzte sich im Wahlkampf stark ein, betrieb keine Effekthascherei mehr wie in früheren Wahlkämpfen und wollte zeigen, dass er der bessere Wahlkämpfer ist als diejenigen, die ihn als Parteichef gestürzt hatten. Am Ende waren die Bürger im Vergleich zum Spitzenduo mit seiner Arbeit zufriedener, aber auch er verblieb bis zur Wahl bei der generellen Beurteilung im negativen Bereich.

In der zweiten Julihälfte gab Kanzlerin Merkel der FPD durch ihr Machtwort zur Beibehaltung des Solidaritätszuschlags über 2019 hinaus die Gelegenheit, in Abgrenzung zum Koalitionspartner und den Oppositionsparteien eine neue Steuersenkungsdebatte zu entfachen und sich im Sinne ihrer Kernklientel zu profilieren. Man machte während der Debatte zwar auch deutlich, dass Steuern generell nur beim Erzielen von Haushaltsüberschüssen gesenkt werden könnten, ging aber u.a. mit der Forderung „Soli abschaffen. Deutschland entlasten“ in den Wahlkampf. Munition für den eigenen Wahlkampf lieferte zudem der politische Gegner: In Reaktion auf die umfangreichen Steuererhöhungsbeschlüsse der Grünen und der SPD konzentrierte die FDP den Wahlkampf unter Rainer Brüderles Ägide in traditioneller Weise auf die ökonomisch motivierten Stammwähler im Mittelstand. Es gelang ihr jedoch nicht, den in den Augen der Wähler verloren gegangenen ,Markenkern' im Bereich der Steuer- und Wirtschaftspolitik wieder zu etablieren. Durch die Stilisierung als einziges Bollwerk gegen Steuererhöhungen geriet zudem die Positionierung auf der gesellschaftspolitischen Konfliktlinie, d.h. das Herausstellen der FDP als Bürgerrechtspartei, in den Hintergrund.

$13 \mathrm{Zu}$ diesen Daten vgl. Forschungsgruppe Wahlen e.V., Politbarometer (div. Ausg.); Forschungsgruppe Wahlen e.V., Bundestagswahl. Eine Analyse der Wahl vom 22. September 2013, Mannheim, 2013, S. 27; Infratest dimap, Wahlreport. Bundestagswahl 22. September 2013, Berlin, 2013, S. 38. 
Auf dem Wahlkonvent am 12. September legte sich die FDP offiziell auf eine Koalition mit der Union fest und schloss alle anderen Koalitionsmöglichkeiten aus. Gleichzeitig setzte sie verstärkt auf die Warnung vor einer Regierungsbeteiligung der Linkspartei. Eine Woche vor der Bundestagswahl bekam der Wahlkampf einen empfindlichen Dämpfer: Bei der bayerischen Landtagswahl fiel die FDP von 8,0 auf 3,3 Prozent und wurde von der Regierungspartei zur außerparlamentarischen Oppositionspartei. Die Parteiführung verbreitete Endzeitstimmung und warb jetzt unverhohlen um Zweitstimmen von taktisch wählenden Unionsanhängern. Im Gegensatz zu früheren Bundestagswahlen, stieß diese Strategie bei der Unionsführung jedoch nach den negativen Erfahrungen aus der Niedersachsenwahl auf keinerlei Gegenliebe, und zudem fielen die Medienkommentare zur Zweitstimmenkampagne teilweise extrem negativ aus. Dennoch wurde in den letzten Tagen vor der Wahl allgemein davon ausgegangen, dass die Partei zwar dramatisch an Zustimmung verlieren würde, es aber gerade noch in den Bundestag schaffen könnte, da alle Umfrageinstitute der FDP in ihren Prognosen Werte zwischen 5 und 6 Prozent voraussagten.

\section{Das Wahlergebnis}

Am Wahlabend wurden die Erwartungen der FDP und ihrer Anhänger bitter enttäuscht: Die Partei stürzte von 14,6 Prozent bei der Wahl 2009 auf 4,8 Prozent ab und schied daher nach 64 Jahren parlamentarischer Repräsentanz aus dem Bundestag aus. Im Westen der Republik erreichte sie 5,2 Prozent (2009: 15,4 Prozent), das höchste Ergebnis konnte sie in ihrer traditionellen Hochburg Baden-Württemberg erzielen. Auch dort reichte es jedoch nur für 6,2 Prozent gegenüber 18,8 Prozent im Jahr 2009. Im Osten erhielt sie insgesamt 2,7 Prozent, die geringste Zustimmung war mit 2,2 Prozent in Mecklenburg-Vorpommern zu verzeichnen, wo sie 2009 knapp 10 Prozent erreicht hatte. Bei der Bundestagswahl 2009 hatte sie von allen anderen Bundestagsparteien Stimmen hinzugewinnen können, wobei der Zuwachs von ehemaligen Unionswählern mit über 1,1 Mio. Stimmen mit Abstand am höchsten war. Diesmal verlor sie an alle anderen Parteien, wobei allein über 2,1 Mio. Wähler per Saldo zur Union abwanderten $^{(14)}$.

Die Verluste der Partei waren in den einzelnen sozialen Gruppen unterschiedlich hoch $^{(15)}$. In den letzten beiden Wahlen wurde die FDP überdurchschnittlich von jüngeren Wählern (bis 44 Jahre) gewählt. Dort fielen jetzt die Verluste überdurchschnittlich aus, so dass sie 2013 von allen Altersgruppen in gleichem Maße gewählt wurde. Verstärkt wurde hingegen die Abhängigkeit der FDP-Wahl vom Bildungsniveau: Je höher die formale Schulbildung der Befragten, desto besser schnitt die Partei ab. Betrachtet man die Wahlentscheidung in den Berufsgruppen, so zeigt sich, dass die Verluste unter den Selbstständigen, d.h. der traditionellen Kernwählerschaft der FDP, am höchsten waren: Hatten 200924 Prozent der Selbstständigen die FDP gewählt, so waren es 2013 nur noch 10 Prozent. 


\title{
Fazit
}

Das Schicksal der FDP zeigt, dass angesichts der gestiegenen Flexibilität der deutschen Wählerschaft selbst eine Partei, die die Geschicke der Bundesrepublik von Anfang an mitgeprägt hat, vor einem dramatischen Absturz nicht geschützt ist. Die FDP hat die Erwartungen ihrer Wähler enttäuscht, ihr Vertrauen verspielt, ihren Markenkern verloren, und sie konnte diese Probleme im Wahlkampf nicht durch eine bei den Wählern positiv bewertete neue Spitzenmannschaft sowie durch überzeugende inhaltliche Angebote auffangen. Ob es ihr unter neuer Führung - Christian Lindner ist vom Parteitag am 7./8. Dezember 2013 zum neuen Vorsitzenden gewählt worden gelingt, das verlorene Terrain zurückzugewinnen, ist jetzt noch nicht abzusehen.

\section{Zusammenfassung}

Der Beitrag analysiert die Entwicklung der FDP von ihrem Wahlerfolg bei der Bundestagswahl 2009 bis zu ihrem Ausscheiden aus dem Bundestag bei der Wahl 2013. Er verdeutlicht, dass personelle und inhaltliche Gründe schon 2010 zu einem beispiellosen Niedergang in der Wählergunst geführt haben. Der damalige Parteivorsitzende und Außenminister Guido Westerwelle geriet in der Öffentlichkeit und innerparteilich immer stärker in die Kritik und die Partei konnte die bei den Wählern geweckten Erwartungen im Bereich ihres,Markenkerns', der Steuer- und Wirtschaftspolitik, nicht erfüllen. In den drei Jahren bis zur Bundestagswahl 2013 gelang es der Partei weder durch eine neue Führungsmannschaft mit Philipp Rösler an der Spitze noch durch inhaltliche Initiativen in verschiedenen Bereichen, das bei den Wählern verloren gegangene Vertrauen zurückzugewinnen.

\begin{abstract}
The article analyzes the development of the FDP from its success at the General Election of 2009 to the withdrawal of the party from the parliament at the General Election of 2013. It shows that already 2010 personal and policy reasons led to an unprecedented decline in popular support. The former party leader and foreign minister Guido Westerwelle was more and more criticised by the public and his party-fellows and the party could not fulfill the expectations which were raised in the range of its core competences: the fiscal and economic policy. In the next three years, neither a new leadership team nor various policy initiatives could regain the confidence of the voters.
\end{abstract}

